\title{
Ambiente de imersão virtual como ferramenta para mudança de paradigma no processo de projeto arquitetônico: da representação à intęração
}

Virtual immersive environment as a tool for paradigm shift in architectural design process: from representation to interaction

\author{
> Ana Paula Baltazar \\ Lagear UFMG, Brasil \\ baltazar.ana@gmail.com \\ > Filipe Silva Gonçalves \\ Lagear UFMG, Brasil \\ filipesg88@gmail.com
}

\author{
> Emidio Dias Maciel e Souza \\ Lagear UFMG, Brasil \\ emidiodmsouza@gmail.com
}

> Luiza Silva Metzker

Lagear UFMG, Brasil

metzkerluiza@gmail.com

\author{
> Mateus Moreira Pontes \\ Lagear UFMG, Brasil \\ mateus.pontes@gmail.com
}

\begin{abstract}
This paper discusses the possibility of a low-cost immersive virtual environment (AIVITS) shifting the paradigm from representation to interaction in architecture design process. It first presents the perspectival paradigm in architecture, initiated in the Renaissance and culminating with the separation between design, building and use, having representation as its main product. Then it discusses the possibility of having representation as a tool, no longer as a paradigm, in a design process based on interaction. It then presents the interface used in the low-cost immersive virtual environment, which instead of representing the final appearance of the building, stimulates people to play with constructive possibilities by means of gestures.
\end{abstract}

Keywords: Immersive environment; Virtual reality; Representation; Interaction; Stereoscopy

\section{Ambientes de Imersão e o Paradigma Perspectivico: o Hiato entre Desenvolvimentos Tecnológicos e Processo de Projeto}

Este artigo apresenta a discussão das possibilidades de um ambiente de imersão virtual de baixo custo como ferramenta para mudança de paradigma no processo de projeto de arquitetura. Inicialmente discute-se o paradigma perspectívico, iniciado no Renascimento, introduzindo a representação como processo de projeto da arquitetura. Segundo Baltazar (2012) o paradigma perspectívico é reforçado ao longo dos séculos culminando com a consagração da separação entre projeto e construção pelo arquiteto Jean-Laurent Legeay (1710-1786), que "preconizava a virtuosidade de uma ideia sobre seu potencial construtivo ... implicitamente sugerindo que o conhecimento de construção não seria responsabilidade do arquiteto" (Pérez-Gómez \& Pelletier, 1997, p.220-221). Assim, o arquiteto passa a projetar um desenho que comunica a forma final do edifício reduzindo o processo de projeto ao paradigma perspectívico. O Modernismo encampa sem questionamentos tal paradigma e a arquitetura contemporânea também reproduz a representação bi e tridimensional em meios bidimensionais, como a prancheta e a tela do computador, sem muito questionar. Segundo Baltazar (2012) a representaçâo deve ser encarada como ferramenta e não como paradigma na arquitetura, o que implica mudar o foco do processo de projeto da representação para a interação, tanto para simular o processo construtivo quanto para ampliar as possibilidades de uso dos espaços. Tal processo pautado na interação e não na representação encontra um campo fértil para seu desenvolvimento nos ditos ambientes de imersão virtual, ainda que esse não seja ainda o foco da grande maioria das pesquisas na arquitetura.

É comum que a arquitetura tome emprestado tecnologias que não são desenvolvidas especificamente para ela, e as adaptem em seu benefício. Contudo, mesmo usando tais tecnologias, os ambientes de imersão criados apenas reproduzem o paradigma perspectívico, focando principalmente na visualização de modelos tridimensionais, ou, em alguns casos, em sistemas híbridos nos quais podem ser visualizados tanto imagens estereoscópicas quanto imagens em duas dimensôes. Um exemplo dessa utilização meramente para visualização perspectívica na arquitetura é a Caverna Digital do Núcleo de Realidade Virtual do LSI (Laboratório de Sistemas Integráveis) na Escola Politécnica da USP. A Caverna Digital desenvolveu pesquisas em várias áreas, incluindo, entre outras, a Medicina, Engenharias (Naval, Civil, Mecânica, Eletrônica, Automobilística) e a Arquitetura, na elaboraçáo e visualização de maquetes virtuais. Apesar de divulgar inicialmente o seu foco em imersão e interação com o ambiente virtual, as pesquisas desenvolvidas na CAVE da USP acabam sendo mais voltadas para visualização. Marcelo Zuffo (2001), colaborador da CAVE da USP, explica em sua tese A Convergência da Realidade Virtual e Internet Avançada em Novos Paradigmas de TV Digital Interativa que o real interesse dentre os vários sistemas de multiprojeção estereoscópica disponíveis é propiciar a imersão total do usuário. Ainda que Zuffo (2001) aposte no conceito de imersão total — situação em que o usuário se sente completamente envolto por outra realidade criada por estímulos computadorizados (Murray, 1997) — isso não se tornou a tônica das pesquisas levadas 
a cabo na CAVE. Por exemplo, um dos trabalhos que propiciam maior imersão, dentre os desenvolvidos na CAVE da USP, simula um voo de asa delta sobre a cidade do Rio de Janeiro, trazendo para o usuário uma experiência fortemente visual, por meio da representaçáo da cidade (Soares at al, 2004). O sistema do Virtual Hang-gliding over Rio de Janeiro é composto por uma asa delta em escala 1:1 de onde o usuário navega pela simulação visual estereoscópica da cidade, complementada por vento (há uma ventoinha no sistema) e som. A maior limitação tecnológica para simular o voo de asa delta é a dificuldade de simular a resistência do ar. Obviamente a ventoinha não é capaz de criar tal sensação e a simulação acaba sendo fortemente visual. Há um limite da tecnologia para que a imersão total de fato aconteça.

Entretanto, mesmo em casos com grandes avanços no desenvolvimento tecnológico, tanto de hardware quanto de software, os ambientes de imersão continuam tendo como foco a visualização tridimensional. Isso pode ser verificado no projeto da CAVE2 (Febretti, 2013), um sistema híbrido desenvolvido pelo EVL (Eletronic Visualization Laboratory) da Universidade de Illinois dedicado à visualização. Apesar de extremamente desenvolvida do ponto de vista tecnológico, com a mais avançada estrutura de hardware em termos de ambientes de imersão atualmente, a CAVE 2 não explora suas potencialidades para desenvolver interfaces que permitam o distanciamento da representação por si só, para assim se tornar um ambiente onde a interatividade seja o principal foco. Atividades relacionadas a diversos campos de atuação são realizadas no ambiente, como por exemplo simulaçóes em larga escala de átomos em ultra alta definição (Reda, 2013). Neste caso são simulados os comportamentos de diversas moléculas e suas reaçóes sem nenhuma participação do usuário, que tem apenas a possibilidade da visualizar o processo em três dimensóes. Também foram feitas pesquisas para a visualização de grandes quantidades de informaçóes em duas dimensões no ambiente (Reda, 2014), algo que tem sua efetividade comprovada para situaçóes de trabalho colaborativo e análise e construçáo de sentido de grandes quantidades de dados. Entretanto essa exploração é puramente visual e não incorpora a discussão sobre interatividade. Por fim, uma das mais recentes pesquisas que utilizam a interface da CAVE 2 está relacionada à visualização e exploração em escala da Estação Espacial Internacional, utilizando dados em tempo real fornecidos pela National Aeronautics and Space Administration (NASA) referentes à localização da estação real, com o intuito de possibilitar ao usuário uma melhor percepção espacial da nave. Desse modo, pode-se perceber que, atualmente, a maior parte das pesquisas que utilizam ambientes de imersão estão voltadas para a representação ou simulação visual. Ainda que isso atenda às necessidades de desenvolvimento de certas áreas de conhecimento, no caso da arquitetura, não propicia um avanço no processo de projeto.

No caso da arquitetura, já desde os anos 60, a discussão sobre processo de projeto e participação aponta a necessidade de um desenvolvimento tecnológico específico para além da mera visualização, com maior possibilidade de engajamento dos usuários em todo o processo, desde a concepçáo espacial e construtiva até entendimento e decisão sobre a construção e possibilidades de uso.
Diversos arquitetos experimentaram de formas diferentes processos alternativos de projeto incluindo o usuário em etapas distintas. Tais arquitetos "eram jovens durante a guerra e ... se envolveram no debate acadêmico no fim dos anos 50, tais como Lucien Kroll, Ralph Erskine, Christopher Alexander, Giancarlo de Carlo, John Turner, Bernard Rudofsky, Hassan Fathy, Walter Segal, N. John Habraken e Yona Friedman, para mencionar apenas alguns" (Lopes at al, 2010). Vale ressaltar que o pós-guerra ofereceu campo fértil para a produçáo participativa, pois a partir do questionamento da produção em massa dos projetos habitacionais convencionais, que representavam um empobrecimento formal, criticava-se o processo de projeto tradicional (separação entre projeto, construção e uso) e os procedimentos construtivos (principalmente a divisão do trabalho). Do ponto de vista teórico-crítico, é exemplar o trabalho de John Chris Jones (1991 e 1992) que discute métodos de projeto e aponta criticamente a sua falha e a de seus colegas fazedores de método, por criarem métodos para arquitetos (baseados na especialização representativa do processo de projeto) sem considerar que todas as pessoas são designers em potencial. Importante também é a crítica feita por Sérgio Ferro (2006) do desenho como instrumento de dominação tanto dos construtores quanto dos usuários. Bryan Lawson (2005) alerta sobre as armadilhas do desenho, indicando a facilidade que os arquitetos têm em definir apressadamente um programa de necessidades e projetar a edificação equacionando tal programa visualmente como se fosse um quebra-cabeça, sem de fato problematizar as relaçóes sociais.

Contudo, os desenvolvimentos tecnológicos, principalmente os voltados para visualização, parecem ignorar os avanços das discussôes sobre processo de projeto e participação. Há um hiato entre as duas linhas de pesquisa que precisa ser superado. Os ambientes de imersão estereoscópicos estáo sendo usados na arquitetura primordialmente seguindo o paradigma perspectívico, ampliando as possibilidades de visualização realista de projetos. Mas não podemos deixar de vislumbrar o uso de tecnologia de baixo custo de forma criativa visando a possibilidade de simulação do desempenho da edificação e de imersão do projetista e do usuário no projeto, começando a voltar o foco do processo de projeto para a interação. Apesar da usual reprodução dos procedimentos formalistas de projeto, a imersão virtual vem surgindo como ferramenta capaz de simular situaçóes presenciais de produção e análise de espaços, possibilitando pensar e antecipar problemas operacionais, perceptivos e técnicos, além de questóes ligadas ao espaço habitado. Além disso, o ambiente impulsiona a prática do projeto colaborativo, capaz de integrar diferentes agentes do processo de produção do espaço, numa lógica de criação coletiva, importante passo na direção de repensar a profissão e o ensino.

\section{Ambiente Virtual de Tecnologia Simplificada da Escola de Arquitetura da UFMG}

O Ambiente de Imersão Virtual de Tecnologia Simplificada (AIVITS), desenvolvido na Escola de Arquitetura da UFMG, foi originalmente elaborado pelo Grupo de pesquisa Estúdio Virtual de Arquitetura (EVA) e está atualmente funcionando no laboratório de ensino RADAMES. O AIVITS pode ser definido como uma 
CAVE (Cave Automatic Virtual Environment) de baixo custo e baixa tecnologia. Usualmente as CAVEs se caracterizam por seu sistema baseado em uma máquina de grande porte ou nos chamados aglomerados de computadores (clusters), e por possuir telas e aparatos de custo elevado. Ao contrário, o AIVITS se caracteriza por sua simplificação, consistindo basicamente de um computador, três projetores e a estrutura de suporte. Esse computador possui três placas de vídeo distintas, conectadas aos projetores, que possuem suporte para a estereoscopia. Esses se encontram nas três gaiolas que configuram a estrutura do ambiente, projetando a imagem nos espelhos refletores, que por sua vez diminuem a distância de projeção refletindo as imagens por trás de uma tela fixada na estrutura. $\mathrm{O}$ ângulo formado entre cada um dos espelhos é de 120 graus, característica distinta das CAVE's tradicionais nas quais a angulação é de 90 graus (Figura 1).

O AIVITS possui suporte tanto para estereoscopia ativa (uso de óculos que flicam segundo a frequência das imagens emitidas para o olho esquerdo e direito, respectivamente) quanto passiva (uso de óculos anaglíficos, vermelho/azul ou vermelho/verde, criando filtros para imagens também com filtros coloridos emitidas para o olho esquerdo e direito, respectivamente).

Devido a seu baixo custo em relação às CAVE's tradicionais, sua simplificação tecnológica e sua própria configuração espacial baseada na lógica da Tenda Digital (Baltazar \& Cabral Filho, 2006), o ambiente tem grande potencial de replicação, o que amplia suas possibilidades de uso tanto na prática da arquitetura, como também no seu ensino, introduzindo o uso de novas tecnologias de forma crítica e incentivando o projeto colaborativo.

Além das questôes de suporte estrutural e de hardware do projeto do AIVITS, o principal desafio foi estabelecer uma conexáo entre a prática tradicional dos arquitetos e estudantes e o ambiente de imersão. Na Escola de Arquitetura da UFMG a grande maioria dos estudantes e professores trabalha com modelos tridimensionais usando o SketchUp, que não produz o par estéreo para visualização e também não facilita a programação de características para interação. Assim, o Unity3D, software amplamente utilizado na

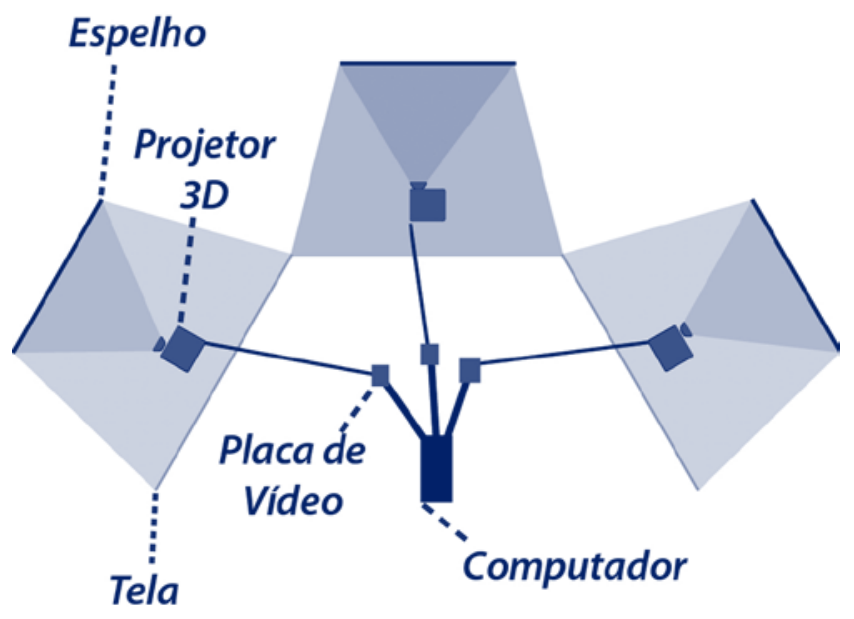

Figura 1: Esquema da estrutura de funcionamento do AIVITS. produção de jogos virtuais, foi escolhido como a primeira interface a ser testada para a importação dos modelos gerados no SketchUp e programação da interação. Isso implica a inclusão do usuário de forma ativa no ambiente de imersão, náo mais apenas visualizando mas interagindo diretamente com os objetos programados. Desta forma pretende-se superar o paradigma perspectívico, abrindo novas possibilidades para repensar a prática arquitetônica.

\section{Unity 30 e as Possibilidades de Imersão Pela Interação}

Uma das principais características do Unity3D é a capacidade de gerar, com o auxílio de scripts e plugins, estereoscopia ativa e passiva. Outro aspecto importante é que, diferentemente da modelagem de um objeto tridimensional somente para visualização, o Unity3D funciona na lógica da criação de um ambiente interativo, com recursos para a criação de luzes, gravidade, propriedades materiais, meios de navegação e qualquer outro parâmetro que se deseje simular de uma maneira interativa e imersiva. Essas características são capazes de fazer com que o usuário perceba um simples modelo tridimensional feito no SketchUp como sendo um ambiente de imersão. Além disso, a operação do software é baseada em scripts e códigos de programação, essenciais para a interação no ambiente de imersão, abrindo inúmeras possibilidades enquanto interface e não meramente modelo $3 \mathrm{D}$. Um exemplo simples já explorado é a inserção de animaçóes interativas no ambiente, como a abertura e fechamento de portas controladas pelo usuário em seu percurso, que sáo realizados a partir de inputs no teclado do computador. Para isso foi sintetizado e disponibilizado um guia de fácil uso que consiste num passo a passo que aborda desde estratégias que facilitam o uso do Unity3D e a compatibilidade entre este e o SketchUp.

Para trabalhar com o modelo do SketchUp no Unity3D recomenda-se fazer a sua exportação no formato .DAE. Outros formatos, como o .3DS, também são compatíveis, porém são arquivos muito pesados e não facilitam a exportação. Ao exportar o modelo, será criada uma pasta com as texturas e componentes usados no SketchUp, que deve ser copiada e colada na pasta Assets no Unity3D. Há várias incompatibilidades que já foram testadas e sistematizadas no guia. Uma das principais incompatibilidades é o fator de escala que do Unity3D não é compatível com o do SketchUp e portanto deve ser alterado. Nos testes, verificou-se que o fator 0.03 para exportação foi o mais bem sucedido para compatibilização da escala. Após seguir todo o passo-a-passo do Guia Unity3D e SketchUp

(http://www.mom.arq.ufmg.br/lagear/wp-content/uploads/2014/09/guia_unity_3d_final.pdf), deve-se arrastar o ícone do arquivo SketchUp para a janela \#Scene no Unity3D e o modelo 3D se torna o cenário do projeto.

\section{Desenvolvimentos Atuais}

Atualmente a possibilidade de simulaçáo de um processo construtivo a partir de módulos importados do SketchUp para a interface do Unity3D está sendo explorada. Para isso a captação de gestos, movimentos e comandos de voz por meio de sensores tem sido usada para que o usuário interaja com o processo construtivo, po- 
dendo manipular componentes construtivos e seus parâmetros no ambiente de imersão para além da mera visualização. Desse modo, estáo sendo testados dois dispositivos capazes de fazer a leitura de movimentos, o Leap Motion e o Kinect. O primeiro consegue fazer a leitura das mãos do usuário, criando um modelo virtual da mesma que reage em tempo real, tornando possível a sua interação com os componentes existentes no ambiente de imersão. Um exemplo é a simulação de um processo construtivo em alvenaria estrutural com finalidade pedagógica, na qual o usuário pode selecionar blocos de concreto ao apontar a mão em sua direção, carregar os blocos ao fechar a mão e movê-la em outra direção, e assentá-los ao abrir a mão novamente (Figura 2). Os componentes são programados com propriedades físicas, como peso, para que quando empilhados reajam estruturalmente de acordo com a gravidade. Ou seja, diferente de um modelo digital simples, nesse ambiente orientado ao objeto, uma parede "cai” caso não seja viável no mundo físico. Assim, o usuário tem uma relação muito mais íntima e intuitiva com a interface do que teria ao apenas visualizar uma estrutura pronta ou utilizar apenas teclado e mouse para interagir.

Há vários estudos que comprovam que a sensação de presença é muito maior quando o usuário interage com o ambiente por meio de gestos do que simplesmente usando mouse e teclado (Usoh, 1999). Apesar desse último ser um tipo de navegação extremamente difundido no âmbito da produção de jogos virtuais, apresenta muitas limitaçôes em se tratando de imersão, já que o usuário simula o processo através de inputs em botôes, e não através de um movimento análogo ao real, como é no caso do Leap Motion. Além disso, destaca-se a possibilidade do uso sensor Kinect (originalmente desenvolvido para o Microsoft Xbox 360) para a captaçáo da forma do corpo do usuário, seus movimentos, deslocamento e comandos de voz, com a vantagem de fazer a captaçáo de corpo inteiro, e não apenas das mãos.

Um fator a favor do uso dessas tecnologias é a sua disponibilidade crescente no mercado e seu custo cada vez menor, posto que parece existir um grande interesse das empresas de tecnologia em

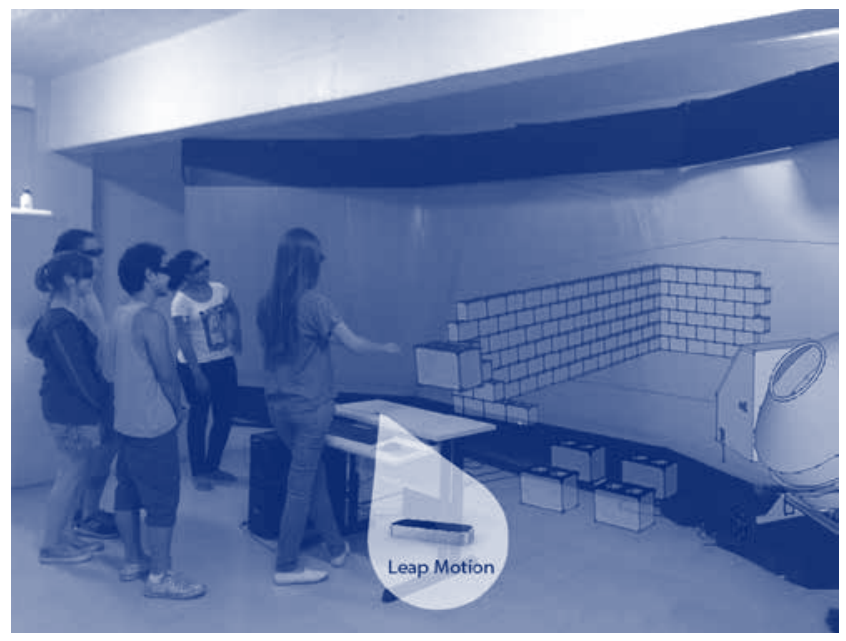

Figure 2: Usuária interagindo via Leap Motion com componentes em canteiro de obras virtual no AIVITS. desenvolver produtos relacionados à realidade virtual, que incluem headsets de realidade virtual, sensores de movimento e voz, TV's UHDTV (8K), networks de alta capacidade de transferência de dados e softwares para a criação de interfaces virtuais, dos quais o Unity3D faz parte. No CineGrid Brasil 2014 (Laurin Herr, comunicação pessoal, 28 de Agosto de 2014) ficou evidente que os desenvolvimentos do mercado apontam cada vez mais para a imersão. Obviamente as tecnologias poderão ser usadas na arquitetura, resta ajuntarmos o desenvolvimento tecnológico com as discussôes sobre processo de projeto, para que a arquitetura não fique refém da reprodução de seus aspectos visuais, ainda que cada vez mais realísticos, e que opere a passagem do paradigma perspectívico (da representação) para o paradigma da interação.

Apontamos, dessa maneira, para a possibilidade de produção de um ambiente de imersão virtual de tecnologia simplificada (AIVITS) e o desenvolvimento de interface usando o Unity3D com modelos do SketchUp. Tal interface indica o potencial do AIVITS para além do paradigma perspectívico.

\section{Agradecimentos}

Os autores gostariam de agradecer as agências de fomento FINEP, CAPES, CNPq e Fapemig e também à Pró-Reitoria de Graduação da UFMG (Programa de Inovação e

Qualidade no Ensino de Graduação), por suas bolsas e financiamentos às pesquisas.

\section{Referências}

Baltazar, A. P. (2012). Além da representaçâo: possibilidades das novas mídias na arquitetura. V!RUS. N. 8: RE:PRE:SENTAR. São Carlos: Nomads.usp. Retrieved from http://www.nomads. usp.br/virus/virus08/?sec $=4 \&$ item $=1 \&$ lang $=$ pt

Baltazar, A. P. \& Cabral Filho, J. S. (2006). Tenda Digital/Digital TENT (Technological Environment for Negotiated Topology) e suas possíveis implicações em contextos sociais. X SIGRADI Congreso Iberoamericano de Gráfica Digital. Santiago: Universidad de Chile, 346-349.

Febretti, A., Nishimoto, A., Thigpen, T., Talandis, J., Long, L., Pirtle, JD, Peterka, T., Verlo, A., Brown, M., Plepys, D., Sandin, D., Renambot, L., Johnson, A. \& Leigh, J. (2013). CAVE2: A Hybrid Reality Environment for Immersive Simulation and Information Analysis. Proceedings of IS\&T / SPIE Electronic Imaging, the Engineering Reality of Virtual Reality. San Francisco, CA.

Ferro, S. (2006). Arquitetura e trabalho livre. Sáo Paulo: Cosac Naify. International Space Station (ISS) and Space Shuttle Discovery. (n.d.) Retrieved from http://www.evl.uic.edu/core.php?mo$\mathrm{d}=4$ \&type $=1$ \&indi $=427$

Jones, J. C. (1991). Designing designing. London: Architecture design and technology press.

Jones, J. C. (1992). Design methods. 2nd edition, New York: Van Nostrand Reinhold.

Lawson, B. (2005). How designers think: the design process demystified. Oxford: Architectural Press.

Lopes, J. M. A., Kapp, S. \& Baltazar, A. P. (2010). Por partes: o novo fundamentalismo participacionista nos programas de 
moradia para os pobres. Silacc 03 - Simpósio ibero-americano de cidade e cultura: novas espacialidades e territorialidades urbanas. São Carlos: EESC-USP.

Murray, J. H. (1997). Hamlet on the Holodeck: The Future of Narrative in Cyberspace. Cambridge: MIT Press.

Pérez-Gómez, A. \& Pelletier, L. (1997). Architectural representation and the perspective hinge. Cambridge: MIT Press.

Reda, K., Knoll, A., Nomura, K., Papka, M.E., Johnson, A. E. \& Leigh, J. (2013). Proceedings of the IEEE Symposium on Large-Scale Data Analysis and Visualization. Atlanta, GA, 59-65.

Reda, K., Offord, C., Johnson, A. \& Leigh, J. (2014). Expanding the Porthole: Leveraging Large, High-Resolution
Displays in Exploratory Visual Analysis. Proceedings of CHI 2014 Conference on Human Factors in Computing Systems. Toronto, Ontario, Canada, 2047-2052.

Soares, L., Nomura, L., Cabral, M., Dulley, L., Guimarães, M., Lopes, R. \& Zuffo, M. K. (2004). Virtual Hang-gliding over Rio de Janeiro. IEEE Virtual Reality Workshop "Virtual Reality for Public Consumption”, IEEE VR, 112-115.

Usoh, M. et al. (1999). Walking >walking-in-place>flying, in virtual environments. SIGGRAPH 99 Computer graphics proceedings, annual conference series. Los Angeles, 359-364.

Zuffo, M. K. (2001) A Convergência da Realidade Virtual e Internet Avançada em Novos Paradigmas de TV Digital Interativa. Escola Politécnica da USP, São Paulo. 